\title{
El empleo de las cooperativas. Un análisis comparativo de sus fases cíclicas y de su grado de sincronización
}

\author{
Mercè Sala Ríos, Teresa Torres Solé, Mariona Farré \\ Perdiguer
}

\section{RESUMEN}

El trabajo realiza un análisis comparativo del comportamiento cíclico del empleo de las cooperativas en España. El periodo de estudio se extiende desde 1995 hasta 2014. El objetivo es estudiar hasta qué punto el ciclo económico de la economía española influye en las variaciones del empleo de las cooperativas y si este muestra un comportamiento distinto al del empleo del conjunto de la economía. Para ello en primer lugar, se caracterizan las fases cíclicas del empleo de las cooperativas, de la actividad económica y del empleo total. En segundo lugar, se evalúa su nivel de sincronización. Por último, se estudia la incidencia de la crisis iniciada en 2007 en la sincronización de los ciclos. Los resultados muestran que el empleo de las cooperativas tiene un comportamiento cíclico relativamente divergente al del ciclo económico y al del empleo total, sin llegar a detectarse un efecto refugio significativo. La crisis ha producido cambios en esta relación, así, las cooperativas se han vuelto más sensibles al ciclo económico y su empleo ha disminuido su resistencia.

PALABRAS CLAVE: Cooperativas, fases cíclicas, puntos de giro, algoritmo de Bry y Boschan, ciclo económico, sincronización cíclica, crisis económica.

CLAVES ECONLIT: E240, E320, J210, J540, L210.

Cómo citar este artículo: SALA, M., TORRES, T. \& FARRÉ, M. (2015): "El empleo de las cooperativas. Un análisis comparativo de sus fases cíclicas y de su grado de sincronización", CIRIECEspaña, Revista de Economía Pública, Social y Cooperativa, 83, 115-141.

Correspondencia: Mercè Sala Ríos, Profesora Titular de Universidad, mercesa@ econap.udl.cat; Teresa Torres Solé, Profesora Titular de Universidad, torres@econap.udl.cat; Mariona Farré Perdiguer, Profesora Titular de Universidad, mariona.farre@econap.udl.cat, Departamento de Economía Aplicada, Universidad de Lleida. 


\title{
L'emploi dans les coopératives. Une analyse comparative de ses phases cycliques et de son degré de synchronisation
}

RÉSUMÉ : Ce document réalise une analyse comparative de l'évolution cyclique de l'emploi dans les coopératives en Espagne. La période d'étude s'étend de 1995 à 2014. L'objectif est de déterminer à quel point le cycle économique de l'économie espagnole influence les variations de l'emploi dans les coopératives et si ce dernier évolue différemment de l'emploi dans l'économie nationale. Tout d'abord, les phases cycliques de l'emploi dans les coopératives, dans l'activité économique et dans l'emploi total sont étudiées. Leur niveau de synchronisation est ensuite évalué. Dans un dernier temps, les répercussions de la crise de 2007 sur la synchronisation des cycles sont analysées. Les résultats indiquent que l'évolution cyclique de l'emploi dans les coopératives est relativement différente de celle de l'activité économique et de l'emploi total. Aucun effet refuge significatif n'a toutefois été détecté. Le crise a modifié cette relation. En effet, les coopératives sont devenues plus sensibles au cycle économique et l'emploi qu'elles génèrent est moins résistant.

MOTS CLÉ : Coopératives, phases cycliques, points de changement, algorithme de Bry et Boschan, cycle économique, synchronisation cyclique, crise économique.

\section{Employment in cooperative societies - A comparative analysis of cyclical phases and synchronization}

\begin{abstract}
This study provides evidence on the relationship between business cycle, employment and cooperative society employment in Spain over the 1995-2014 period. The aim was to examine to what point the cooperative employment cycle is led by the business cycle and whether it shows different cyclical behavior to that of Spanish employment as a whole. The turning points of the cooperative employment, economic activity and total employment cycles were identified; the synchronization level between series was assessed; and the effects of the 2007 crisis on cycle synchronization were studied. The results show little synchronization between the phases of the employment cycle of cooperative societies and of the business cycle, although significant counter-cyclical behavior was not found. However, the crisis has increased their linkage: cooperative employment has become more sensitive to the business cycle and its capacity to create or maintain jobs has decreased.
\end{abstract}

KEY WORDS: Cooperative societies, cyclical phases, turning points, Bry and Boschan algorithm, business cycle, cyclical synchronization, economic crisis. 


\section{EL EMPLEO DE LAS COOPERATIVAS. UN ANÁLISIS COMPARATIVO DE SUS FASES CÍCLICAS Y DE SU GRADO DE SINCRONIZACIÓN}

\section{1.- Introducción}

La actual crisis económica ha incrementado la atención de la literatura sobre fórmulas alternativas de organización empresarial. En este sentido, el derrumbe de muchas empresas con su efecto perverso sobre el empleo, ha reabierto el debate acerca de la capacidad de las empresas de la Economía Social para ofrecer una alternativa viable a ciertos ámbitos de negocio que parecían reservados a la economía capitalista tradicional. La Ley 5/2011 de 29 de Marzo de Economía Social señala que la Economía Social engloba el conjunto de actividades económicas y empresariales que llevan a cabo aquellas entidades que persiguen el interés general económico, social, o ambos. Las entidades que forman la Economía Social son las cooperativas, las sociedades laborales, las mutualidades, los centros especiales de empleo, las empresas de inserción, las cofradías de pescadores, las asociaciones y las fundaciones. El estudio que presentamos se centra en las cooperativas porque es la principal figura de la Economía Social. En términos relativos vemos que representan el 64,84\% del número de empresas e instituciones de la Economía Social y aportan el 74,39\% del empleo¹.

El elevado volumen de desempleo que ha alcanzado la economía española suscita la duda sobre si existen ciertos mecanismos que sean más resistentes a los cambios en la actividad económica y que permitan mantener el empleo o reducir su destrucción. Bajo esta óptica, la pregunta que nos planteamos es ¿son las cooperativas uno de estos mecanismos? Lo primero que llama la atención es que dentro de las cooperativas el factor trabajo alcanza una importancia significativa. Además, existe una amplia literatura que reconoce a las cooperativas una mayor resistencia en el empleo. Entre las principales causas que se esgrimen destacamos las siguientes. Ante caídas de la actividad económica es conocido el proceso de transformación de empresas con problemas económicos o al borde del cierre en cooperativas, lo que preserva el empleo². Asimismo, el aumento del desempleo conlleva que una de las posibles respuestas de los desempleados sea luchar contra el paro estructural creando sus propias empresas (Calderón y Calderón, 2012a; Lejarriaga y Martín, 2010; Grávalos y Pomares, 2001). Autores como Tarazona y Albors (2005), Lampel et al. (2012) o Mcquaid et al. (2013) remarcan el mayor compromiso de los trabajadores con la organización. Los empleados están más dispuestos a cambiar sus condiciones de trabajo, priorizan el trabajo por encima del capital y muestran mayor ini-

1.- Los datos corresponden al primer trimestre de 2014 y se refieren a cooperativas, sociedades laborales, empresas de inserción y centros especiales de trabajo. La fuente es la Confederación Empresarial de Economía Social (CEPES): http://www.cepes.es/tipos_de_entidades.

2.- Para un análisis reciente del fenómeno véase por ejemplo, CECOOP-CICOPA Europe (2013) o el monográfico sobre Emprendimiento y Economía Social de CIRIEC-España (2013). Por su parte la Confederación Empresarial Española de la Economía social (CEPES), en el actual escenario de crisis sigue apostando por estas transformaciones al promover medidas de emprendeduría en la Economía Social que incluyen como escenario emprendedor la posibilidad de transformar empresas en crisis en cooperativas: $h$ ttp://www.cepes.es/pagina_cepes=44. En este sentido Melián y Campos (2010) ensalza la figura del emprendedor social porque incorpora en su proyecto objetivos empresariales y fines sociales, genera empleo estable, profesionaliza la gestión y fomenta el desarrollo local. 
ciativa cuando se trata de encontrar formas de reducir los costos y mejorar la relación con los clientes. También se constata que su participación en el ámbito directivo asegura su motivación y como apuntan Park et al. (2004) o Ben-Ner (1988) la coincidencia de intereses entre empresa y trabajadores ayuda a mitigar los posibles conflictos ${ }^{3}$. Calderón y Calderón $(2012 a, b)$ reconocen la mayor flexibilidad de las cooperativas para ajustarse vía horas trabajadas más que vía número de trabajadores. Esto confiere mayor estabilidad laboral y junto a elementos como la igualdad de género, no discriminación o inclusión incrementa la calidad del empleo (Calderón y Calderón, 2012b; Roelants et al., 2012). La mayor estabilidad en el trabajo facilita la inversión en capital humano y la mejora de las habilidades de los trabajadores, lo cual a su vez revierte en los buenos resultados de la empresa (Park et al., 2004). Por otra parte, se observa que sus estrategias de toma de decisiones son más propensas a introducir oportunidades para la incorporación del input empleo y a buscar un equilibrio entre el capital humano y las oportunidades de negocio (Lampel et al., 2014). Las cooperativas son más selectivas a la hora de contratar y más reacias a la hora de despedir, lo que reduce el incentivo para expandir las operaciones simplemente para aprovechar las oportunidades de las fases expansivas pero también las hace menos vulnerables a las contracciones (Lampel et al., 2012).

El importante papel que ha jugado el empleo de las cooperativas sobre la economía ha llevado también a desarrollar un interesante debate sobre su relación con el ciclo económico. A grandes rasgos, las investigaciones sobre el tema pueden agruparse en dos grandes líneas. En la primera se enmarcan trabajos que encuentran una relación significativa entre el empleo de las cooperativas y el ciclo económico. Dentro de ella por un lado, encontramos estudios que reconocen a las cooperativas un comportamiento contra-cíclico que les lleva a ser una herramienta defensiva ante periodos contractivos y ejercer un efecto refugio. Véase por ejemplo, Guerreri et al. (1992), Cornforth y Thomas (1995), Demoustier (2000), Grávalos y Pomares (2001), Román (2014) Monzón (2012) Calderón y Calderón (2012b). Por otro lado, están aquellos que matizan la existencia de movimientos contra-cíclicos. Así, Sala et al. (2014) llegan a la conclusión que en las fases contractivas las cooperativas presentan relativamente menores pérdidas de empleo y Díaz y Marcuello (2010) que el empleo cooperativo presenta menor dependencia que el conjunto del empleo ante las variaciones a la alza y a la baja del PIB, aunque en ambos estudios los resultados no permiten corroborar un comportamiento contracíclico. Pérotin (2006) señala que si bien la creación de cooperativas es contra-cíclica no es tan evidente que también lo sea la salida y para Ben-Ner (1988) el patrón contra-cíclico existe aunque se ve en parte compensado porque en épocas expansivas también se crean cooperativas y su supervivencia es vulnerable a las épocas contractivas. Por último, dentro de esta primera línea de investigación, encontramos estudios que analizan el papel que ejercen diversas variables a la hora de explicar la naturaleza, la intensidad y el signo de la relación entre el empleo de las cooperativas y el ciclo económico. Variables como por ejemplo, el corto o largo plazo (Ben-ner, 1988; Díaz-Foncea y Marcuello,

3. Lampel et al. $(2010,2012)$ indican que una clara limitación de las empresas propiedad de los empleados se basa en su propio éxito. El vínculo que sienten los empleados entre su esfuerzo y el rendimiento de la empresa les lleva a trabajar mejor y a conseguir mejores resultados. Ello conlleva en ocasiones el crecimiento de la firma, lo que dificulta mantener dicho vínculo y que los trabajadores sigan anteponiendo los intereses de la firma a los propios. El efecto positivo de la participación de los trabajadores es mayor en empresas de pequeño tamaño y va desvaneciéndose cuando la empresa alcanza mayores dimensiones (Bayo-Moriones y Larraza-Kintana, 2009). 


\section{EL EMPLEO DE LAS COOPERATIVAS. UN ANÁLISIS COMPARATIVO DE SUS FASES CÍCLICAS Y DE SU GRADO DE SINCRONIZACIÓN}

2014); la consideración del conjunto de la economía o de las regiones (Clemente et al., 2008, 2009); la importancia de las cooperativas dentro del territorio (Díaz-Foncea y Marcuello, 2014); los cambios en el escenario político, normativo o en la estructura productiva, (Román, 2014). La segunda línea de investigación recoge aquellos trabajos que no encuentran una interacción significativa ente el empleo de las cooperativas y el ciclo económico. Entre ellos podemos destacar estudios como Aldrich y Stern (1983), Conte y Derek (1984), Russell y Hanneman (1992), Staber (1993) o Podivinsky y Stewart (2007).

El estudio que presentamos profundiza en el análisis comparativo de las fases cíclicas del empleo de las cooperativas con las de la actividad económica y las del empleo del conjunto de la economía. El objetivo es estudiar hasta qué punto el ciclo económico de la economía española influye en las variaciones del empleo de las cooperativas y si este muestra un comportamiento distinto al del empleo del conjunto de la economía. El periodo de estudio se extiende desde 1995 hasta 2014. A fin de alcanzar tal objetivo avanzaremos a través de dos líneas de análisis. En primer lugar, caracterizaremos las fases cíclicas del empleo de las cooperativas, la actividad económica y el empleo total, y nos ocuparemos del nivel de sincronización de las tres variables. En segundo lugar, analizaremos la incidencia de la crisis iniciada en 2007 en la sincronización de los ciclos.

Así pues, el trabajo se organiza de la siguiente forma. En el segundo apartado se presentan los indicadores, las fuentes estadísticas y la metodología seguida a lo largo del estudio. El tercer apartado se ocupa de los resultados en relación a la caracterización del ciclo y la sincronización. El cuarto evalúa los efectos de la crisis sobre el nivel de sincronización. El quinto expone las principales conclusiones.

\section{2.- Indicadores, fuentes estadísticas y metodología}

El indicador que se ha utilizado para aproximar las fluctuaciones cíclicas de la economía española es el PIB real trimestral corregido de estacionalidad. La fuente estadística ha sido el INE. Los datos sobre el empleo de la economía española proceden también del INE. Por lo que respecta a los datos de las Cooperativas se han obtenido de los Datos Estadísticos de Economía Social del Ministerio de Empleo y Seguridad Social. Son datos trimestrales de su número de trabajadores registrados en el Régimen General de la Seguridad Social y en el Régimen Especial de Autónomos. Todas las series han sido transformadas en log. El periodo que entra en el estudio es desde el primer trimestre de 1995 (1995:1) hasta el tercero de 2014 (2014:3).

La definición de ciclo económico más extendida es la de ciclo de negocios (business cycle) de Burns y Mitchel (1946). Según los autores, un ciclo se compone de fluctuaciones en los niveles de la 
actividad económica en fases expansivas y recesivas que se repiten de forma no recurrente. Después de la II Guerra Mundial y sobretodo debido a los largos periodos de crecimiento estable de los años sesenta, las caídas de la actividad económica no fueron ni muy frecuentes ni muy duraderas, lo que propició que los estudiosos del tema empezaran a interesarse por lo que Mintz (1969) denominó ciclo de crecimiento (growth cycle), esto es, las fluctuaciones en torno a un crecimiento tendencial. Tales fluctuaciones pueden analizarse como fluctuaciones respecto a la tendencia (ciclo de desviaciones) 0 como fluctuaciones en las tasas de crecimiento (ciclo de tasas de crecimiento) ${ }^{4}$.

En este estudio se trabaja con el ciclo de desviaciones. Para calcular la desviación respecto a la tendencia o el componente cíclico de las tres series, PIB, empleo de la economía española y trabajadores de las cooperativas, se ha aplicando el filtro de Hodrick-Prescott (HP) a las series trimestrales. La elección de HP se ha realizado básicamente porque al ser simétrico no produce movimientos de fase, porque aproxima bien a un filtro ideal cuando se utiliza un $\lambda=1.600$ para datos trimestrales y porque es un método operacional.

Dentro del ciclo se definen dos fases cíclicas, la fase expansiva y la contractiva, para acotarlas el primer paso es determinar los puntos de giro (picos y valles) de las series. Un pico se define como el momento en el tiempo inmediatamente anterior a una disminución de la serie y un valle como el momento previo al crecimiento de la serie. Así, la contracción es el lapso que media entre el pico (P) y el valle $(\mathrm{V})$ y la expansión es el lapso que media entre el valle $(\mathrm{V})$ y el pico $(\mathrm{P})$. La duración del ciclo es el tiempo que transcurre entre un pico y el siguiente o entre un valle y el siguiente.

Los picos y los valles de los componentes cíclicos del empleo de las cooperativas, el PIB y el empleo total se obtendrán siguiendo el algoritmo de Bry y Boschan (1971), adoptado a datos trimestrales según Harding y Pagan (2002). El software utilizado es el denominado BUSY, desarrollado por la Comisión Europea (Fiorentini y Planas, 2003). El algoritmo exige el cumplimiento de una serie de restricciones relacionadas con la duración y la amplitud de las fases y del ciclo completo que sintetizamos en tres reglas fundamentales. En primer lugar, un máximo/mínimo local se define como el punto más alto/bajo entre los dos trimestres predecesores y antecesores a su posición. Es decir $y_{t}$ es un pico en el tiempo $t$ si $y_{t}$ es el $\max \left(\mathrm{y}_{\mathrm{t}-2} \ldots \mathrm{y}_{\mathrm{t}+2}\right)$ y es un valle si $\mathrm{y}_{\mathrm{t}}$ es el $\min \left(\mathrm{y}_{\mathrm{t}-2} \ldots \mathrm{y}_{\mathrm{t}+2}\right)$. En segundo lugar, un ciclo siempre debe completarse, por lo que un pico debe ir seguido de un valle y viceversa, con una duración mínima de la fase cíclica de tres trimestres ${ }^{5}$. Por último, la duración mínima de un ciclo completo es de cinco trimestres 6 .

4.- El ciclo de crecimiento se asocia con la idea de aceleraciones y desaceleraciones de la actividad económica mientras que el concepto de business cycle se asocia más con la de auges y recesiones (Arango et al., 2007). El número de ciclos de crecimiento es mayor que el de los business cycles, dado que las desaceleraciones en el crecimiento no siempre suponen disminuciones en el nivel de las series. Entonces, el business cycle incorpora el ciclo de crecimiento. Como indican Zarnowitz y Ozyildirim (2006) todas las recesiones implican desaceleraciones, pero no todas las desaceleraciones conllevan recesiones. 


\section{EL EMPLEO DE LAS COOPERATIVAS. UN ANÁLISIS COMPARATIVO DE SUS FASES CÍCLICAS Y DE SU GRADO DE SINCRONIZACIÓN}

A partir de los puntos de giro identificados se iniciará el análisis del des/acoplamiento de las tres variables. En muchos trabajos para aproximar el des/acoplamiento se recurre a metodologías que utilizan como indicador el diferencial de crecimiento. De forma general se concluye que existe desacoplamiento si dicho diferencial es positivo y tiende a crecer con el tiempo. Este tipo de metodologías no resultan adecuadas a nuestro estudio puesto que no toman en consideración las fases cíclicas de las variables. Si llegáramos a la conclusión de que el empleo de las cooperativas crece más que el PIB y además la brecha se amplia se debería concluir que dicho crecimiento se produce con independencia de la fase cíclica del PIB. Nuestro objetivo pretende ligar los co-movimientos con las fases cíclicas, es decir, pretende conocer lo que en la literatura de ciclos económicos se conoce como la sincronía entre las series. Si dos variables muestran sincronía cíclica implica que las etapas del ciclo, expansión y contracción, se dan en momentos del tiempo similares, es por ello que la metodología adecuada al objetivo del trabajo es analizar el grado de sincronización. Esta metodología al ligar los co-movimientos a las fases cíclicas, detecta conductas pro- contra-cíclicas y nos permitirá llegar a conclusiones sobre si en el período de estudio se observa o no un comportamiento diferencial del empleo de las cooperativas en relación al ciclo económico y al empleo de la economía española. Como ventaja adicional, los resultados serán de utilidad para futuros estudios que persigan explicar los factores o causas determinantes del grado de acoplamiento cíclico entre las series o los efectos que sobre el mismo pueden causar shocks de carácter endógeno o exógeno.

El análisis de la sincronización se iniciará calculando un indicador para todo el período. De acuerdo con Harding y Pagan (2002), obtendremos el índice de concordancia (I) que precisamente entiende la sincronización como la proporción de tiempo en la que dos variables $(i, j)$ se encuentran en la misma fase:

$$
I_{i j}=T^{-1}\left[\sum_{t=1}^{T}\left(S_{i t} S_{j t}\right)+\sum_{t=1}^{T}\left(1-S_{i t}\right)\left(1-S_{j t}\right)\right]
$$

donde $S_{i t}\left(S_{j t}\right)$ es una variable binaria que toma el valor 1 cuando la variable $i(j)$ está en expansión y cero cuando está en recesión y T es el número de observaciones.

El índice resulta adecuado principalmente por dos motivos. Primero porque proporciona una información fácil de interpretar. El índice se mueve entre uno y cero, la unidad indica perfecta concordancia cíclica y cero perfecta inexistencia de concordancia o perfecta concordancia contra-cíclica. Segundo

5.- Bry y Boschan (1971) proponen una longitud de la fase de 6 meses, sin embargo, en la mayoría de trabajos que utilizan datos trimestrales se establece en tres trimestres. La decisión se fundamenta en el hecho de que está comúnmente aceptado que una recesión implica dos trimestres consecutivos de crecimiento negativo del PIB (véase Wecker, 1979), al incorporar la restricción de tres trimestres se exige que una vez reconocida la recesión esta se extienda al menos otro trimestre. El razonamiento nos parece adecuado por lo que hemos fijado dicha duración como longitud mínima de la fase, además al trabajar de igual forma que muchos otros estudios se facilitan posibles análisis comparativos.

6. - Como indican Harding y Pagan (2002) la duración mínima de 15 meses establecida por Bry y Boschan (1971) es compatible con 405 trimestres, dependiendo del mes en el que se produzca el punto de quiebre y de las magnitudes relativas de los meses dentro del trimestre. Es por ello que se han calculado los puntos de giro con 4 y con 5 trimestres sin que ello haya variado los puntos de giro que son los que presentamos en el cuadro 1 y los gráficos 1 a 3. 
porque aunque las series estén integradas sus resultados no se ven afectados (Harding y Pagan, 2002). Sin embargo, tiene una limitación importante que es la imposibilidad de conocer si los co-movimientos son estadísticamente significativos. Precisamente para salvar esta dificultad estimaremos el coeficiente de correlación $(\rho)$ entre $S_{i t}$ y $S_{j t}$, usando el método generalizado de momentos según la metodología propuesta por Harding y Pagan (2006). Partimos de la condición de los momentos:

$$
\mathrm{E}\left[\left(\sigma s_{i}^{-1}\left(S_{i t}-\mu s_{i}\right) \sigma s_{j}^{-1}\left(S_{j t}-\mu s_{j}\right)\right)-\rho s\right]=0
$$

donde os y us son respectivamente la media y la desviación típica de las series $S_{i t}$ y $S_{j t}$. El estimador que se genera es:

$$
\frac{1}{T} \sum_{t=1}^{T}\left[\left(\hat{\sigma} s_{i}^{-1}\left(S_{i t}-\hat{\mu} s_{i}\right) \hat{\sigma s} s_{j}^{-1}\left(S_{j t}-\hat{\mu} s_{j}\right)\right)-\hat{\rho} s\right]=0
$$

El proceso de estimación utilizado es el de Newey y West (1987) (HAC) y pesos de Bartlett que resulta consistente a la presencia de correlación serial y heteroscedasticidad. La significancia estadística puede contrastarse entonces usando la t-ratio. Una t-ratio significativa y positiva indica sincronización pro-cíclica. En cambio, una t-ratio significativa y negativa indica asimetría contra-cíclica.

Con los cálculos anteriores tendremos una visión conjunta del grado de sincronización dentro del período. Sin embargo, para poder conocer el efecto de la crisis sobre el grado de simetría cíclica nos interesa desglosar temporalmente la sincronización cíclica. Para ello calcularemos un índice de asimetría (IA) que cuantifica la tendencia de la sincronización de los ciclos (Larsson et al., 2009 quienes citan a Hassler, 2003). Se normalizará el componente cíclico de las series (Y), mediante la desviación estándar $(\sigma): Y^{\star}=Y / \sigma$. El índice entre dos variables se define como:

$$
I A_{i j t}=\left|Y_{i t}^{*}-Y_{j t}^{*}\right|
$$

La evidencia de que la series i y jpresentan sincronización significativa en el tiempo se analizará mediante la ecuación ajustada por mínimos cuadrados a la tendencia lineal del IA:

$$
I A_{i j t}=\beta_{0}+\beta_{1} L T_{t}+\varepsilon_{t}
$$




\section{EL EMPLEO DE LAS COOPERATIVAS. UN ANÁLISIS COMPARATIVO DE SUS FASES CÍCLICAS Y DE SU GRADO DE SINCRONIZACIÓN}

Donde $L T=1,2,3, \ldots t$ y $t=$ número de trimestres. Los resultados se interpretarán de la siguiente forma:

- $\beta_{1}<0$ y t-ratio significativo: existe evidencia de simetría temporal significativa en las series

- $\beta_{1}<0$ y t-ratio no significativo: existe evidencia de simetría temporal no significativa en las series

- $\beta_{1}>0$ y t-ratio significativo: existe evidencia de asimetría temporal significativa en las series

- $\beta_{1}>0$ y t-ratio no significativo: existe evidencia de asimetría temporal no significativa en las series

- IA=0: las dos variables presentan plena simetría temporal en los ciclos

\section{3.- Resultados: caracterización del ciclo y sincronización}

Iniciamos el análisis de los resultados caracterizando las fases cíclicas. El cuadro 1 recoge los puntos de giro y los Gráficos 1 a 3 la evolución de los componentes cíclicos de los trabajadores de las cooperativas (TCS), del PIB y del empleo de la economía española (EM), que son las tres variables sobre las que deseamos estudiar su comportamiento cíclico. EI PIB y el EM presentan tres ciclos completos de valle a valle y dos de pico a pico. Las cooperativas en cambio, alcanzan un único ciclo completo de valle a valle y dos ciclos completos de pico a pico. Los puntos de giro se muestran más homogéneos entre el componente cíclico del PIB y el EM que entre el componente cíclico de estas dos series y el TCS. Así, vemos que el empleo de las cooperativas no sufrió el proceso contractivo de mediados de los noventa que sí se observa en el PIB y en el EM. Además hay retardos significativos en el momento de alcanzar el pico y el valle en las fases que se extienden de finales de los noventa hasta la llegada de la crisis financiera a mediados de 2007. La crisis parece romper esta divergencia y acercar el comportamiento de las series.

De lo dicho se desprende que en la fase de mayor crecimiento de la economía española, el TCS mostró un comportamiento bastante divergente al del PIB y el EM. En cambio, la crisis de 2007 implicó un mayor grado de similitud en las fases cíclicas de las tres variables y una disminución de los retardos en los picos y valles. El análisis del grado de sincronización permitirá dar mayor robustez a estos resultados. 
Cuadro 1. Turning Points PIB, EM y TCS

\begin{tabular}{|l|c|c|c|c|c|}
\hline \multicolumn{2}{|c|}{ PIB } & \multicolumn{2}{c|}{ EM } & \multicolumn{2}{c|}{ PCS } \\
\hline & Vico & Pico & Valle & Valle \\
\hline $2000: 4$ & $1997: 3$ & & $1998: 1$ & $1999: 4$ & \\
\hline $2007: 4$ & $2004: 3$ & $2000: 3$ & $2004: 1$ & & $2002: 3$ \\
\hline $2011: 4$ & $2009: 3$ & $2007: 3$ & $2010: 1$ & $2007: 4$ & $2010: 2$ \\
\hline
\end{tabular}

FUENTE: Busy y elaboración propia.

\section{Gráfico 1.}

Componente cíclico TCS

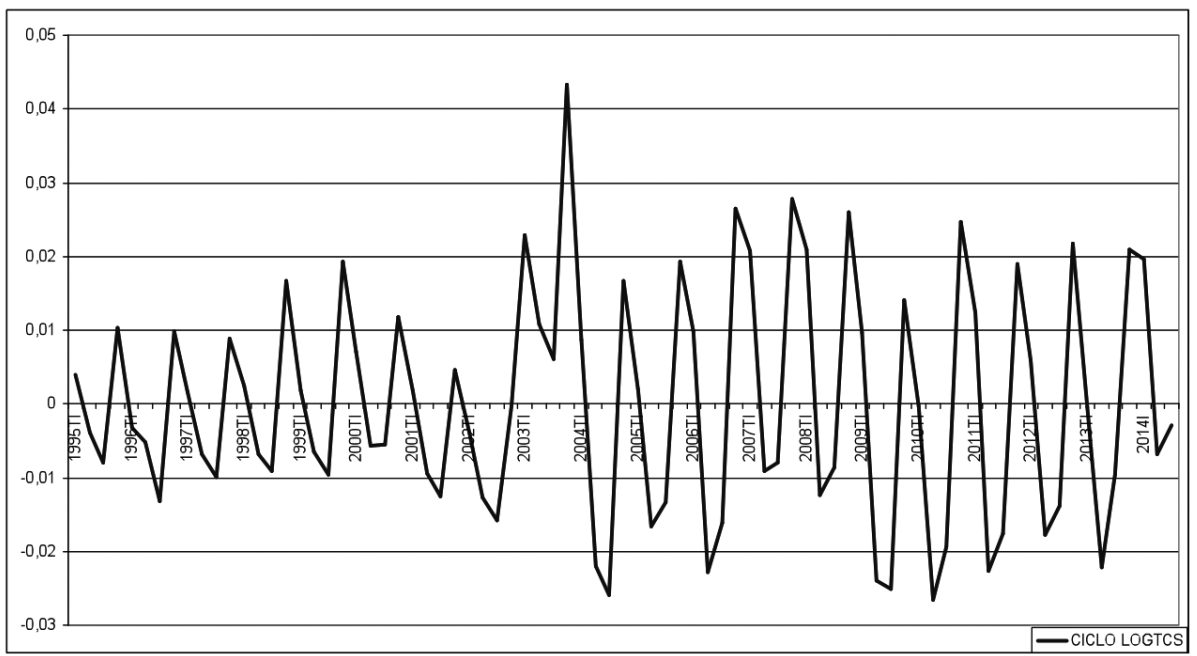

FUENTE: Busy. 
EL EMPLEO DE LAS COOPERATIVAS. UN ANÁLISIS COMPARATIVO DE SUS FASES CÍCLICAS Y DE SU GRADO DE SINCRONIZACIÓN

Gráfico 2. Componente cíclico PIB

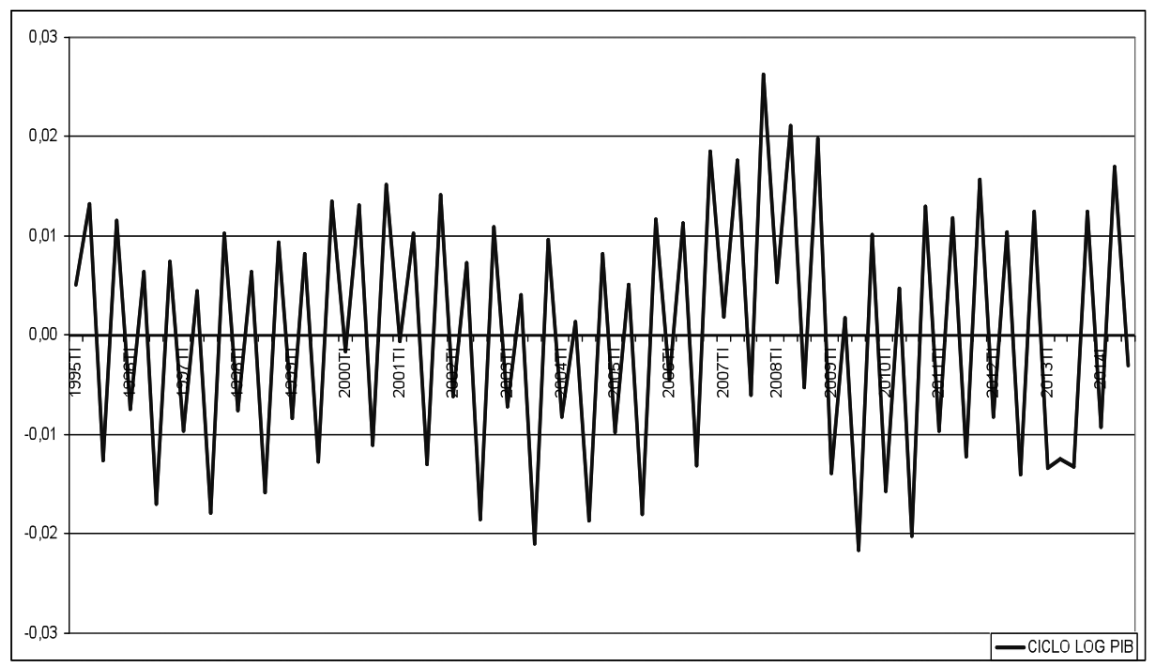

FUENTE: Busy.

\section{Gráfico 3. Componente cíclico EM}

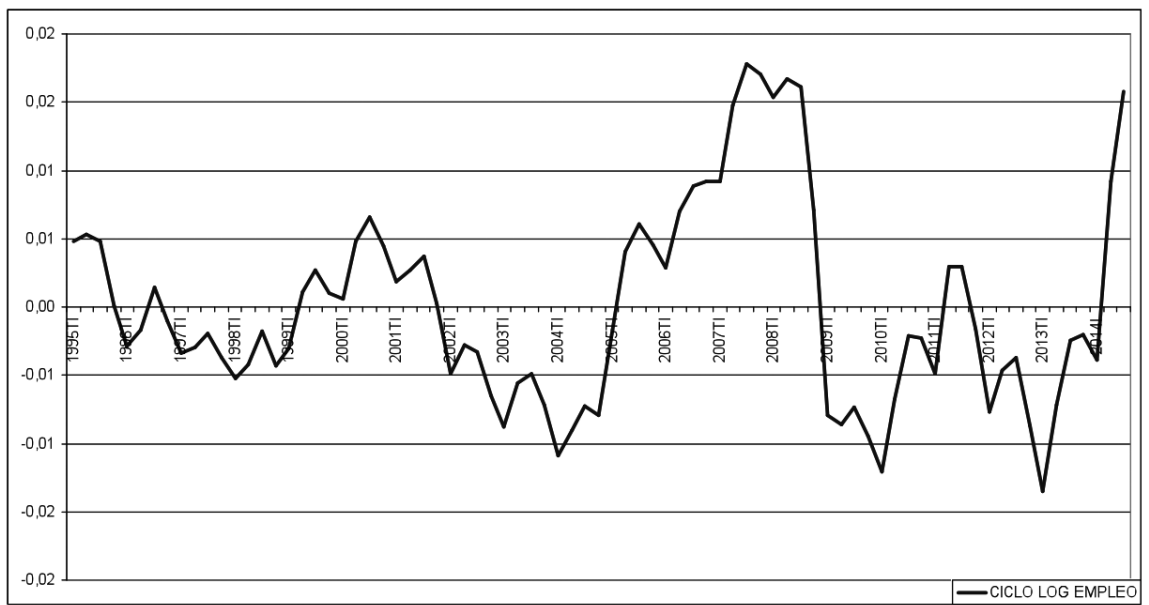

FUENTE: Busy. 
Para cuantificar el grado de sincronización en las fases cíclicas, el primer índice que se ha calculado es el índice de concordancia (I) que para todo el periodo, alcanza los valores del cuadro 2. El índice de concordancia entre el PIB y el EM presenta un valor relativamente elevado. En cambio, los datos revelan un nivel relativamente menor de sincronización entre el PIB y el TCS. Lo que confirma que el empleo de las cooperativas muestra un comportamiento cíclico relativamente divergente respecto al del ciclo económico y pone de manifiesto que existe menor sincronía cíclica entre el número de trabajadores de las cooperativas y el PIB que entre este y el EM.

\section{Cuadro 2. Índice de concordancia}

\begin{tabular}{|l|l|}
\hline PIB- TCS & 0,544 \\
PIB-EM & 0,886 \\
EM-TSC & 0,557 \\
\hline
\end{tabular}

FUENTE: Elaboración propia.

Nos interesa ver hasta qué punto los grados de simetría/asimetría cíclica obtenidos en el cuadro 2 son significativos. Para ello se ha estimado el coeficiente de correlación (r) usando el método generalizado de momentos expresado en (3). El cuadro 3 nos ofrece los valores de la $t$-student y del nivel de significación.

\section{Cuadro 3. t-Student y significación. Estimación $\rho$}

\begin{tabular}{|l|r|r|}
\hline \multicolumn{1}{|c}{$\boldsymbol{t}$-Student } & \multicolumn{1}{c|}{ Sign. } \\
\hline PIB-TCS & $-0,788549$ & 0,4328 \\
PIB-EM & 5,031678 & 0,0000 \\
EM-TCS & 0,836908 & 0,4052 \\
\hline
\end{tabular}

FUENTE: Elaboración propia.

Según los datos del cuadro 3 existe una correlación ampliamente significativa entre el EM y el PIB. En cambio, el TCS no alcanza una correlación significativa ni con el PIB ni con el EM. Es más, el signo de la $t$-student de la estimación entre el PIB y el TCS es negativo. De lo que se deduce un comportamiento contra-cíclico respecto a la actividad económica, aunque no significativo. 


\section{EL EMPLEO DE LAS COOPERATIVAS. UN ANÁLISIS COMPARATIVO DE SUS FASES CÍCLICAS Y DE SU GRADO DE SINCRONIZACIÓN}

Este leve comportamiento contra-cíclico de las cooperativas en relación al PIB ise debe al comportamiento de las fases expansivas (contractivas) de las cooperativas, del PIB o de ambos? Para dar respuesta a esta pregunta el análisis no puede realizarse por la misma vía que nos ha llevado a los resultados del cuadro 3 . El estimador del coeficiente de correlación $(\rho)$ entre $S_{i t}$ y $S_{j t}$, expresado en (3) no puede calcularse puesto que la desviación estándar $\left(\sigma_{S}\right)$ de la serie binaria $S_{i}\left(S_{j}\right)$ es cero cuando para la variable TCS (PIB) sólo se considera su fase expansiva (contractiva). Es por ello que hemos procedido a calcular el coeficiente de correlación entre el componente cíclico del PIB y el del TCS, en las fases expansivas (contractivas) del TCS y en las fases expansivas (contractivas) del PIB. EI resultado es el que presenta el cuadro 4.

\section{Cuadro 4. Estimación $\rho$ de los componentes cíclicos del PIB y el TCS}

\begin{tabular}{|l|r|}
\hline Fase expansiva PIB-TCS & 0,455 \\
Fase contractiva PIB-TCS & 0,339 \\
Fase expansiva TCS-PIB & $-0,144$ \\
Fase contractiva TCS-PIB & 0,339 \\
\hline
\end{tabular}

FUENTE: Elaboración propia.

Los datos indican la existencia de un leve comportamiento contra-cíclico en las fases expansivas del TCS que en cambio no se detecta en las fases contractivas del PIB. Aunque a priori pueda parecer contradictorio, la explicación radica en los retardos en alcanzar los puntos de giro que muestran ambas variables. El cuadro 5 recoge el cálculo de los retardos (adelantos) medios en los picos y los valles del TCS en relación al PIB. Como puede observarse, los puntos de giro del empleo de las cooperativas se adelantan a los de la actividad económica. De la información de los cuadros 4 y 5 podemos concluir que las cooperativas son pro-cíclicas con las contracciones de la economía (0,339 de correlación), sin embargo, superan dicha fase cuatro trimestres antes de que lo haga la actividad económica (cuadro 5). Las cooperativas son capaces de crear empleo en períodos en los que la economía sigue en contracción, lo que explica el leve comportamiento contra-cíclico detectado en sus fases expansivas. Un comportamiento contra-cíclico que sin embargo, no alcanza un valor significativo que permita atribuirles un efecto refugio (cuadro 3).

El resultado no se aleja de los obtenidos por otros estudios centrados en periodos similares al nuestro. Destacamos los trabajos de Díaz y Marcuello (2010), para los años 1995 a 2008, y de DíazFoncea y Marcuello (2014), para 1994 a 2007, en ambos se concluye que el empleo de las cooperativas no genera un efecto refugio sobre el conjunto del empleo de la economía española. Díaz y Marcuello (2010) reconocen sin embargo que el empleo de las cooperativas es menos sensible a las 
fases del ciclo económico. Es decir, mientras en las fases expansivas las cooperativas muestran menos dinámica en la creación de empleo en periodos de contracción la destrucción también es menor.

Por lo que se refiere a las fases expansivas del PIB y las contractivas del TCS vemos en el cuadro 4 que presentan coeficientes de correlación positivos. En los períodos en los que la economía está en expansión las cooperativas tienden a crear empleo y en las fases en las que las cooperativas pierden empleo suelen venir acompañadas de contracciones en el PIB. Las variaciones en la actividad económica influyen en las variaciones del empleo de las cooperativas. Ahora bien, según los datos del cuadro 5, el TCS se adelanta 2 trimestres a la hora de alcanzar el pico. A medio plazo, la expansión de la actividad económica viene acompañada de pérdidas en el empleo de las cooperativas, aunque el efecto no sea tan intenso como para generar un comportamiento contra-cíclico. La buena situación económica ayuda a mejorar los resultados y el nivel de empleo de las empresas tradicionales lo que incentiva la transformación de cooperativas en empresas no sociales que ven disminuir su número y sus trabajadores. Estamos ante lo que se denomina efecto sustitución (Díaz-Foncea y Marcuello, 2014). Para la economía española estos autores constatan la presencia de este efecto en el largo plazo y dentro de aquellas Comunidades Autónomas con mayor peso del empleo cooperativo sobre el empleo total. Como en nuestro caso, el fenómeno no se detecta en el corto plazo.

\section{Cuadro 5. Retardo medio (trimestres)}

\begin{tabular}{|l|l|}
\hline & $\operatorname{TCS}^{(1)}$ \\
\hline Picos & $+2,5$ \\
Valles & +4 \\
\hline
\end{tabular}

(1) TCS respecto a PIB. - (+) implica retardo (adelanto).

FUENTE: Busy y elaboración propia.

\section{4.- Los efectos de la crisis sobre el nivel de sincronización}

Hasta ahora nos hemos ocupado de la sincronización cíclica sin tener en cuenta su evolución temporal. En este apartado, para poder ver la influencia de la crisis de 2007, analizaremos la evolución de la sincronización mediante el índice de asimetría (IA). Los gráficos 4 a 6 muestran la evolución del IA a la que se ha ajustado la tendencia lineal estimada, debajo de cada gráfico consta la ecuación ajustada y el estadístico-t. El indicador de asimetría muestra que con el paso del tiempo se han agu- 
dizado las divergencias cíclicas entre las tres series $\left(\beta_{1}>0\right)$, además, el estadístico-t es significativo, por lo tanto, hay evidencia de asimetría temporal significativa en las series. En el conjunto del periodo ha existido un proceso de desacoplamiento entre las fases cíclicas del empleo de las cooperativas con respecto al PIB y al conjunto del empleo, lo cual está en línea con los resultados de los indicadores de sincronización obtenidos.

\section{Gráfico 4. IA PIB/TCS}

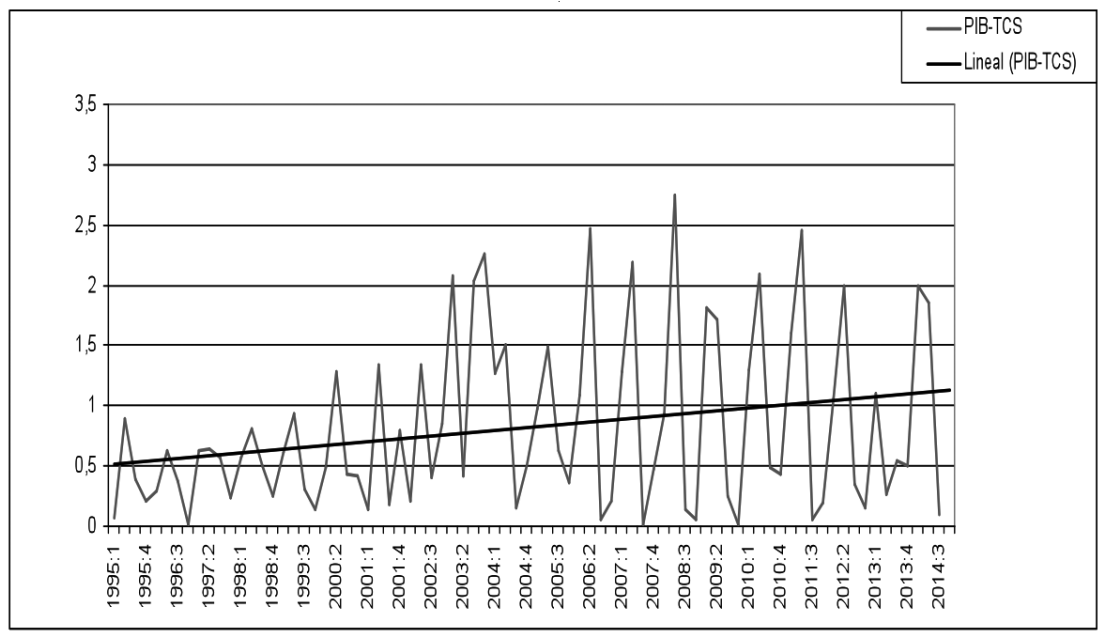

$\mathrm{Y}=0,0078 \mathrm{X}+0,5059$

$\mathrm{t}=2,261^{\star *}$ 
Gráfico 5. IA PIB/EM

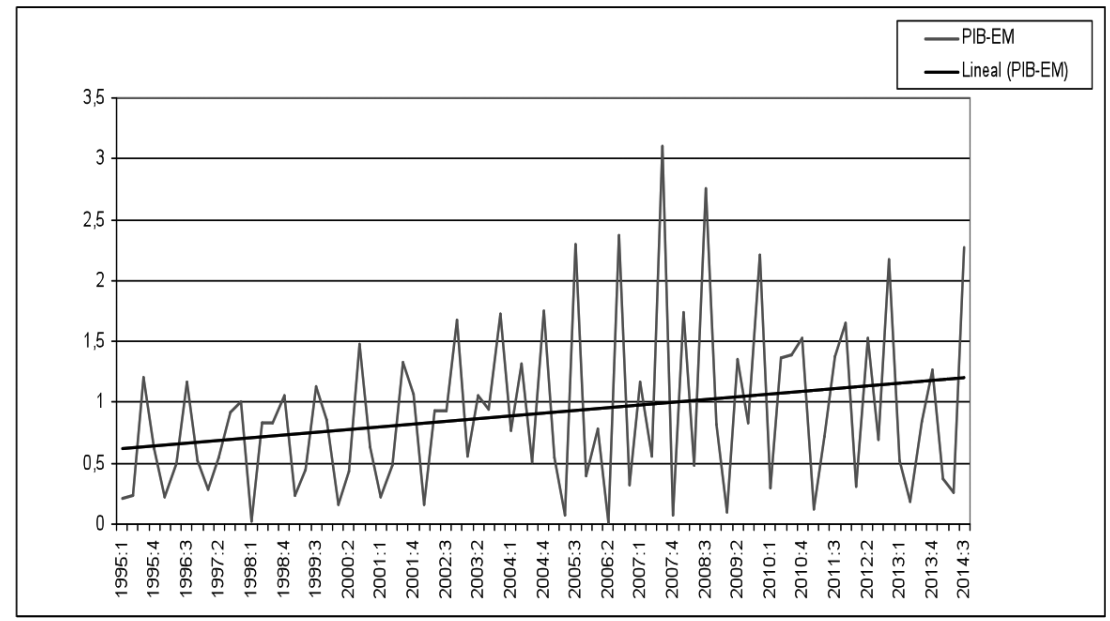

$Y=0,0074 X+0,6122$

$\mathrm{t}=2,267^{\star \star}$

\section{Gráfico 6. IA EM/TCS}

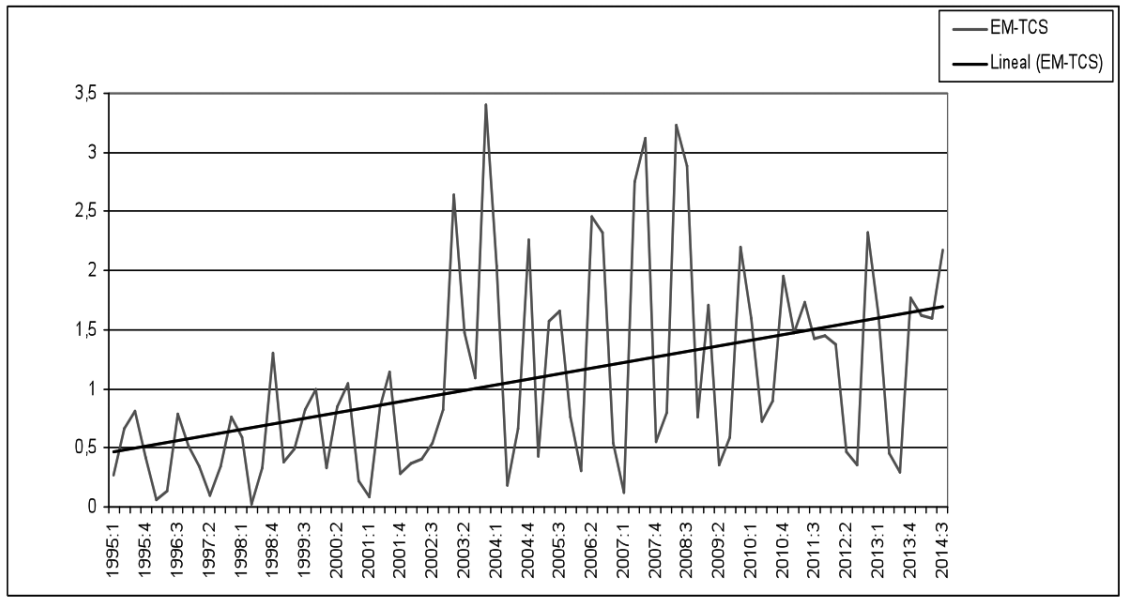

$Y=0,0158 X+0,4464$

$\mathrm{t}=4,1361^{*}$

** Sign al $5 \%$.

*Sign al 1\%.

FUENTE: Busy y elaboración propia.

CIRIEC-España, Revista de Economía Pública, Social y Cooperativa № 83/2015, pp. 115-141

ISSN: 0213-8093 
El fuerte impacto de la crisis iniciada a mediados del año 2007 nos lleva a preguntar si la tendencia del IA descrita puede estar influenciada por las cifras de los años de crisis. En este sentido recordemos que al ocuparnos de los puntos de giro se intuía que la crisis había disminuido la divergencia cíclica entre el PIB, el EM y el TCS. Para ver lo que ello significa sobre el grado de asimetría presentamos los gráficos 7 a 12. En ellos aparece de nuevo el índice de asimetría pero esta vez para dos subperiodos, por un lado 1995:1-2007:3 y por otro 2007:1-2014:3. Las estimaciones de $\beta_{1}$ y el estadístico-t acompañan de nuevo a los gráficos.

\section{Gráfico 7. IA PIB/TCS 1995:1/2007:4}

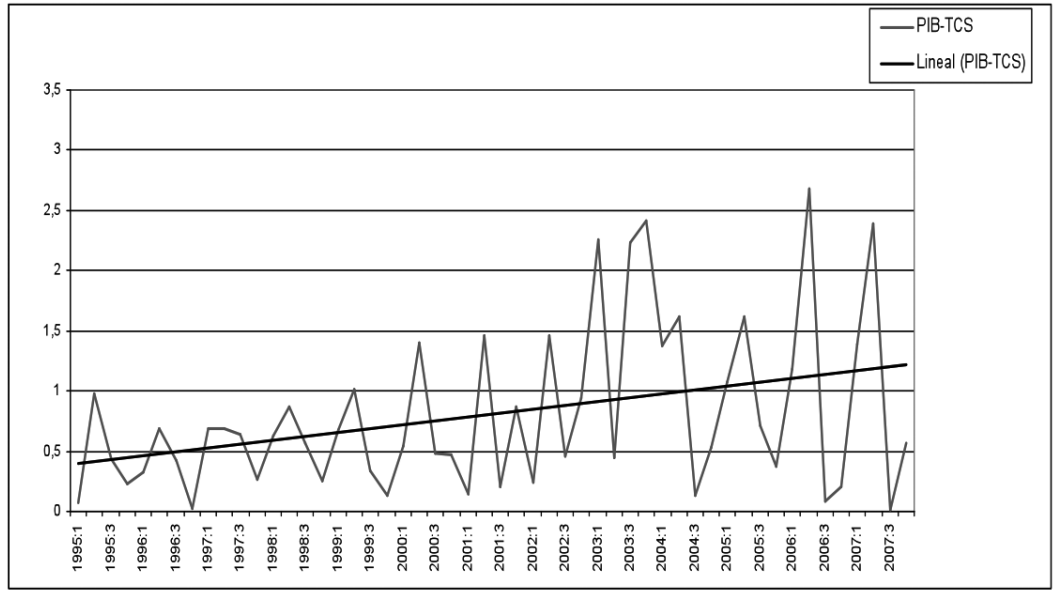

$Y=0,0161 X+0,3788$

$\mathrm{t}=2,717^{\star *}$ 
Gráfico 8. IA PIB/TCS 2007:1/2014:3

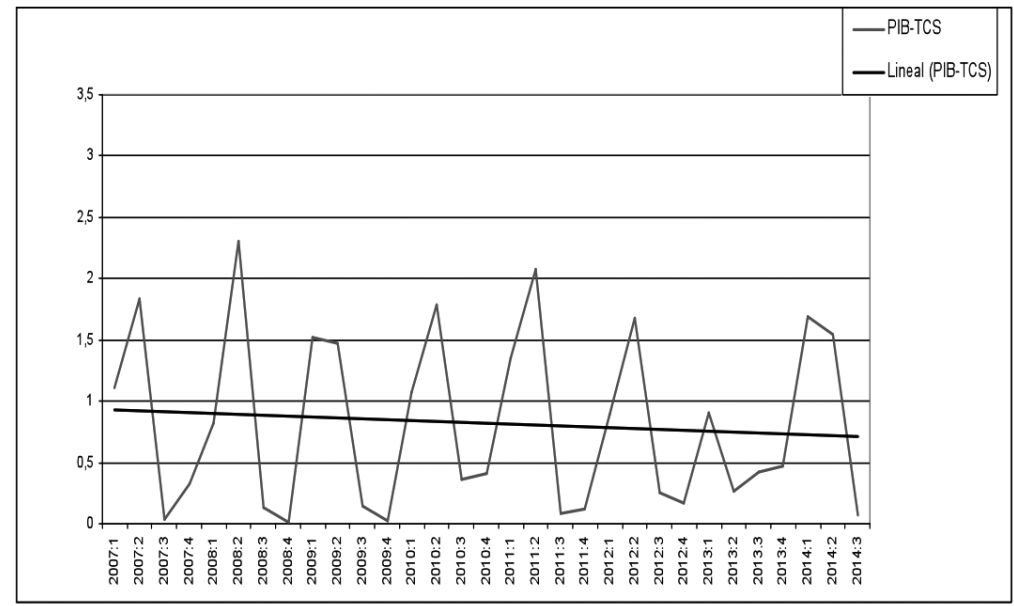

$Y=-0,0072 X+0,9326$

$\mathrm{t}=-0,023$

\section{Gráfico 9. IA PIB/EM 1995:1/2007:4}

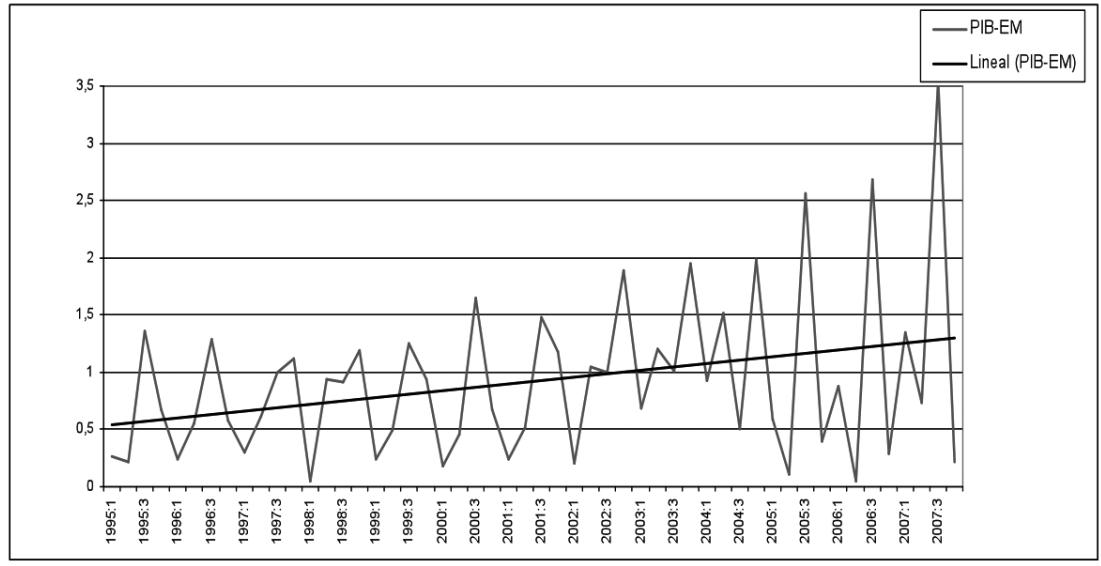

$Y=0,0148 X+0,5287$

$\mathrm{t}=2,317^{\star \star}$ 
EL EMPLEO DE LAS COOPERATIVAS. UN ANÁLISIS COMPARATIVO DE SUS FASES CÍCLICAS Y DE SU GRADO DE SINCRONIZACIÓN

\section{Gráfico 10. IA PIB/EM 2007:1/2014:3}

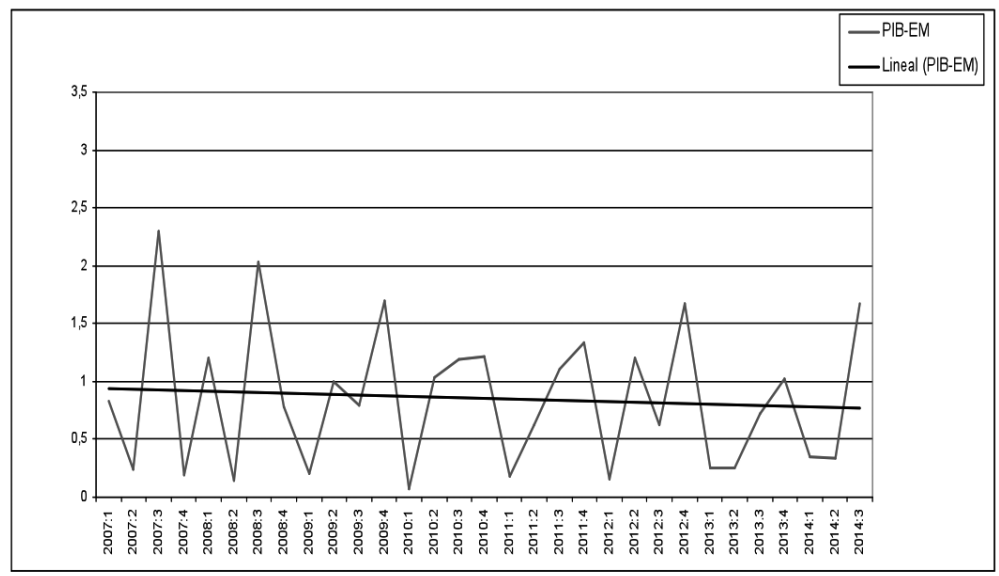

$Y=-0,0056 X+0,9411$

$\mathrm{t}=-0,096$

\section{Gráfico 11. IA EM/TCS 1995:1/2007:4}

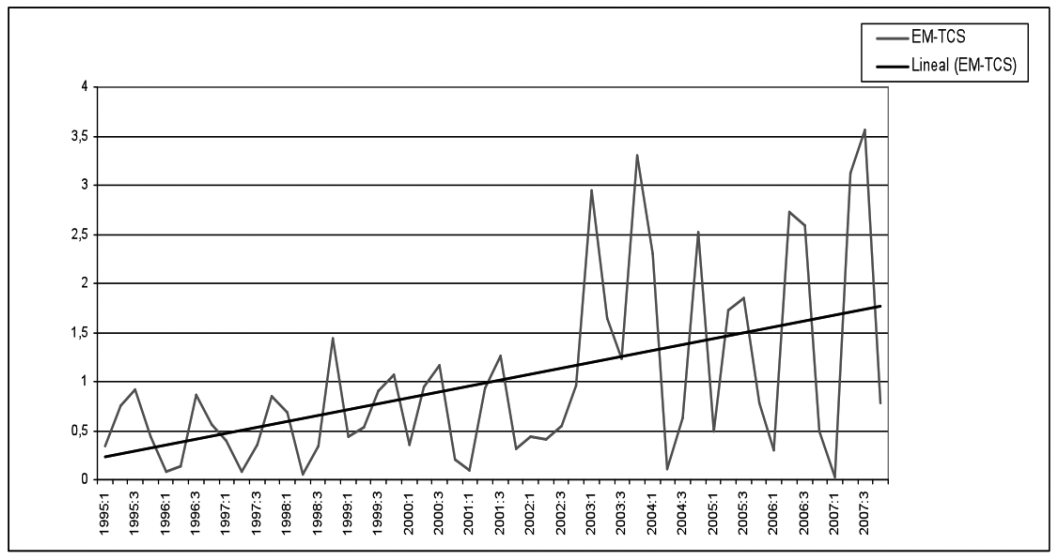

$Y=0,0302 X+0,1993$

$\mathrm{t}=4,005^{\star}$ 
Gráfico 12. IA EM/TCS 2007:1/2014:3

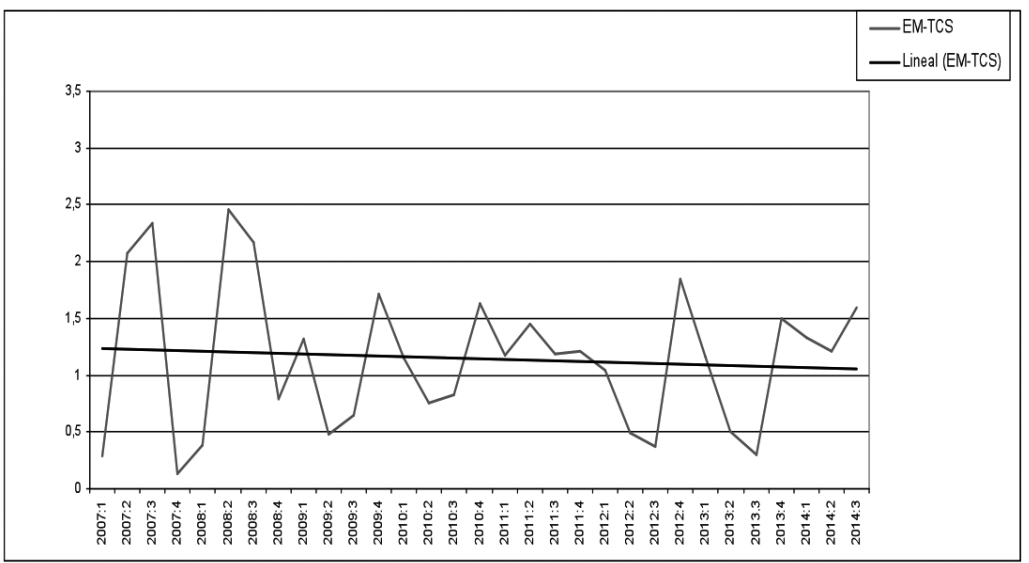

$Y=-0,006 X+1,2406$

$\mathrm{t}=-0,282$

** Sign al $5 \%$.

*Sign al $1 \%$

FUENTE: Busy y elaboración propia.

El período anterior a la crisis sigue mostrando asimetrías significativas sin cambios remarcables con respecto al conjunto del período. En cambio, observamos que las pendientes positivas de los gráficos 7, 9 y 11 se convierten en negativos para los años de crisis (gráficos 8, 10 y 12). La crisis ha incrementado la simetría de las tres series, es decir, con la crisis el empleo de las cooperativas ha incrementado su grado de acoplamiento con el ciclo económico español y con el empleo total.

Para ver la posible repercusión de la crisis sobre el leve efecto refugio que habíamos detectado, en el cuadro 6 se ha calculado la t-student del cuadro 3 pero ahora para dos subperiodos 1995-2006 y 2007-2014, aunque los resultados no sean estadísticamente robustos, dado el número reducido de observaciones, sí que permiten detectar si ha habido cambios de orientación con la crisis. El valor hallado para el sub-periodo 1995-2006 es $(-2,277706)$, mucho más elevado que el $(-0,788549)$ del conjunto del período y que ya recogía el cuadro 3 . El valor de los años de crisis deja de ser negativo y pasa a ser positivo. En los años de crisis el empleo de las cooperativas se ha vuelto más sensible a las fases del ciclo y el efecto refugio ha desaparecido. 


\section{Cuadro 6. t-Student y significación. Estimación $\rho$ por sub- periodos}

\begin{tabular}{|l|ccc|}
\hline & $\begin{array}{c}t \text {-Student } \\
\text { Todo el periodo }\end{array}$ & $\mathbf{1 9 9 5 - 2 0 0 6}$ & $\mathbf{2 0 0 7 - 2 0 1 4}$ \\
\hline PIB-TCS & $-0,788549$ & $-2,277706$ & 0,644036 \\
\hline
\end{tabular}

FUENTE: Elaboración propia.

Los resultados deben tomarse con cierta cautela y precisan de ciertas matizaciones. Así, en primer lugar, los valores negativos de $\beta_{1}$ de los gráficos 8 y 12 no son significativos, es decir, existe evidencia de simetría temporal pero no significativa en las series. En segundo lugar, en términos absolutos las pendientes negativas de estos gráficos son más suaves que las positivas de los gráficos 7 y 11 , lo cual indica que el grado de acoplamiento de los años de crisis es menos intenso que el desacoplamiento que se había producido entre 1997 y 2007, aunque se ha producido en un periodo de tiempo mucho más corto.

En tercer lugar, como puede verse en el gráfico 13, la pérdida de empleo en las cooperativas ha sido relativamente menor que la del conjunto de la economía española. Como indican Monzón y Chaves (2012) para la Economía Social en su conjunto, todo indica que las cooperativas resistieron mejor que el sector privado la primera embestida de la crisis, sin embargo, desde finales de 2008 y especialmente en el año 2009, empezaron a perder empleo. Ahora bien, los efectos sobre el empleo no han sido tan intensos como los del conjunto de empleo. 


\section{Gráfico 13. Variación interanual del empleo de las cooperativas y del empleo total (2006-2014)}

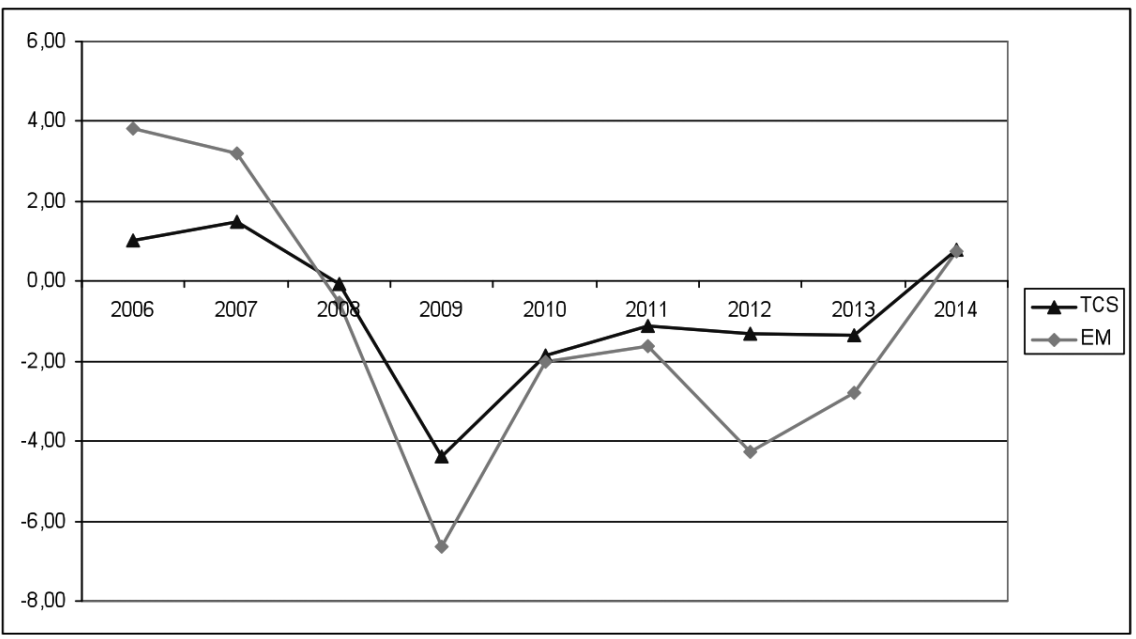

2014: tres primeros trimestres.

FUENTE: Elaboración propia.

De lo dicho podemos concluir que si bien en los años de crisis se ha incrementado el impacto del ciclo económico sobre el empleo de las cooperativas, estas han seguido mostrando una resistencia relativamente mayor que el empleo del conjunto de la economía. Los resultados coinciden con los obtenidos por Calderón y Calderón (2012a) derivados de un análisis de la Muestra Continua de Vidas Laborales de la Seguridad Social. Para estos autores la causa fundamental del comportamiento diferencial del empleo en las cooperativas en esta crisis radica en dos procesos simultáneos como son la transformación de empresas tradicionales en cooperativas y la creación de este tipo de empresas por parados. 


\section{EL EMPLEO DE LAS COOPERATIVAS. UN ANÁLISIS COMPARATIVO DE SUS FASES CÍCLICAS Y DE SU GRADO DE SINCRONIZACIÓN}

\section{5.- Conclusiones}

El trabajo propone caracterizar las fases cíclicas del empleo de las cooperativas y comparar su comportamiento con el de la actividad económica y el empleo del conjunto de la economía española dentro del periodo 1995-2014. Asimismo, revisa la incidencia de la crisis iniciada en 2007 en la sincronización de los ciclos. El interés del estudio radica en ver hasta qué punto el empleo de las cooperativas es o no sensible a la evolución del ciclo económico de la actividad económica y si muestra un comportamiento distinto al del empleo del conjunto de la economía.

Para alcanzar tal objetivo se ha procedido a determinar las fases cíclicas de las series del PIB real de la economía española, del empleo y del empleo de las cooperativas para a continuación evaluar el grado de sincronización cíclica. Los principales resultados a los que se ha llegado son los que se exponen a continuación.

En primer lugar, el empleo de las cooperativas tiene un comportamiento cíclico relativamente divergente al del ciclo económico y al del empleo total. Ello se constata por un lado, porque el PIB de la economía española sufrió un proceso contractivo a mediados de los noventa que en cambio, no afectó a las cooperativas y que desde finales de los noventa hasta la llegada de la actual crisis, hay retardos destacables en los puntos de giro. Por otro lado porque el índice de concordancia entre el PIB y el empleo de las cooperativas revela un nivel relativamente bajo y no significativo de sincronización.

En segundo lugar, el coeficiente de correlación entre el componente cíclico del PIB y el del empleo de las cooperativas es negativo aunque no estadísticamente significativo. Este resultado avala un cierto comportamiento contra-cíclico que según nuestro análisis deriva de las fases expansivas del empleo en las cooperativas. Las cooperativas tienen capacidad de crear empleo en periodos en los que la actividad económica se encuentra en su fase contractiva, aunque su efecto no es lo suficientemente importante para dotar a estas instituciones sociales de un efecto refugio significativo.

En tercer lugar, los resultados indican que a medio plazo la expansión en la actividad económica influye en las variaciones del empleo de las cooperativas. El crecimiento económico genera un efecto sustitución que repercute negativamente en el empleo de las cooperativas. El buen momento de la economía ejerce un efecto positivo sobre el empleo total, recordemos que ambas variables están ampliamente sincronizadas, produciéndose un desplazamiento del empleo de las cooperativas. Así, a medio plazo la expansión de la actividad económica viene acompañada de pérdidas en el empleo de las cooperativas, aunque el efecto no sea tan intenso como para generar un comportamiento contracíclico. 
Por último, la crisis ha supuesto un acoplamiento entre el empleo de las cooperativas y el ciclo económico del PIB y del empleo. Las cooperativas se han vuelto más sensibles a las fases del ciclo y ha desaparecido el efecto refugio. La intensidad de la crisis y su carácter sistémico ha minado en parte las razones que confieren al empleo de las cooperativas una mejor posición para hacer frente a las fases contractivas de la economía. Sin embargo, los efectos sobre el empleo son relativamente menos intensos de los que ha sufrido el empleo del conjunto de la economía.

Para finalizar, es necesario apuntar que el trabajo no va más allá de analizar el posible comportamiento diferencial del empleo de las cooperativas respecto a la actividad económica y al conjunto del empleo de la economía española para unos años en concreto, sin que las conclusiones pueda generalizarse ni extrapolarse a otros periodos temporales. Cabe señalar además, que no se detiene en el análisis de las causas explicativas de dicho comportamiento diferencial. En la introducción revisábamos las principales razones que según la literatura confieren mayor resistencia al empleo de las cooperativas. Al llegar a este punto se nos plantea cuáles de dichas razones y qué otras están detrás de los resultados que hemos presentado para el período 1995-2014 y cuáles se han debilitado con la crisis. Dado el interés que suscita el tema, es una línea de investigación que queda abierta para un estudio futuro.

\section{6.- Bibliografía}

ALDRICH, H. \& STERN, R. (1983): "Resource Mobilization and the Creation of US Producer's Cooperatives, 1835-1935", Economic and Industrial Democracy, 4 (3), 371-406.

ARANGO, L.E., ARIAS, F. \& FLÓREZ, L.A. (2007): "Cronología de los ciclos de crecimiento recientes en Colombia", Borradores de Economía, 464, Banco de la República.

BAYO-MORIONES, A. \& LARRAZA-KINTANA M. (2009): "Profit-sharing plans and affective commitment: Does the context matter?", Human Resource Management, 48 (2), 207-226.

BEN-NER, A. (1988): "The life-cycle of worker-owned firms in market economies. A theorical analysis", Journal of Economic Behavior and Organization, 10 (3), 287-313.

BRY, G. \& BOSCHAN, Ch. (1971): Cyclical analysis of time series: selected procedures and computer programmes, National bureau of Economic Research, New York.

BURNS, A.F. \& MITCHELL, W.C. (1946): Measuring Business Cycles, NBER, Studies in Business Cycle, Columbia University Press, New York. 


\section{EL EMPLEO DE LAS COOPERATIVAS. UN ANÁLISIS COMPARATIVO DE SUS FASES CÍCLICAS Y DE SU GRADO DE SINCRONIZACIÓN}

CALDERÓN, B. \& CALDERÓN, M.J. (2012a): "Cómo afrontan la crisis las cooperativas en España: comparativa de trayectorias laborales a partir de la Muestra continua de Vidas Laborales", CIRIECEspaña, Revista de Economía Pública, Social y Cooperativa, 76, 5-26.

CALDERÓN, B. \& CALDERÓN, M.J. (2012b): "La calidad del empleo de las entidades de la economía social en el período de crisis", Ekonomiaz, 79, 30-57.

CECOOP-CICOPA Europe (2013): Business Transfers to Employees under the Form of a Cooperative in Europe. Opportunities and Challenges, CECOOP-CICOPA Europe.

CLEMENTE, J., DÍAZ, M. \& MARCUELLO, C. (2008): Estudio sobre las cooperativas y sociedades laborales en España: creación de empleo y contribución al desarrollo económico, Proyecto de Investigación, memoria Final, Dirección General de la Economía Social, del Trabajo Autónomo y del Fondo social Europeo, Ministerio de Trabajo e Inmigración.

CLEMENTE, J., DÍAZ, M. \& MARCUELLO, C. (2009): "Sociedades cooperativas y sociedades laborales en España: estudio de su contribución a la creación de empleo y al crecimiento económico", REVESCO, Revista de Estudios Cooperativos, 98, 35-69.

CLEMENTE, J., DÍAZ, M., GONZÁLEZ, M.A., MARCUELLO, C. \& NACHAR, P. (2014): "Evolución del comportamiento estratégico de las cooperativas versus empresas capitalistas: una aproximación descriptiva al efecto crisis", REVESCO, Revista de Estudios Cooperativos, 116, 77-102.

CONTE, M. \& DEREK, C.J. (1984): "In Search of a Theory of Formation for U.S. Producer Cooperatives: Tests of Alternative Hypotheses". In: B. Dennis, Proceedings of the Thirty Seventh Annual Meeting of IRRA, 377-84.

CORNFORTH, C. \& THOMAS, A. (1995): "Les cooperatives de travail au Rouyaume- Uni. Declin ou croissance?", Revue de Etudes Cooperatives, Mutualistee et Associatives, 255 (53), 17-22.

CHAVES, R. \& SAVALL, T. (2013): "La insuficiencia de las actuales políticas de fomento de cooperativas y sociedades laborales frente a la crisis en España", REVESCO, Revista de Estudios Cooperativos, 113, 61-91.

DEMOUSTIER, D. (2000): "Analysis of employment". In: The enterprises and organizations of the third system: a strategy Challenger for employment, CIRIEC-International, 36-56.

DÍAZ, M. \& MARCUELLO, C. (2010): "Impacto económico de las cooperativas. La generación de empleo en las sociedades cooperativas y su relación con el PIB", CIRIEC-España, Revista de Economía Pública, Social y Cooperativa, 67, 23-44.

DÍAZ-FONCEA, M. \& MARCUELLO, C. (2014): "The relation between total employment and cooperative employment: a convergence and causality analysis", Spatial Economic Analysis, 9 (1), 71 92.

FIORENTINI, G. \& PLANAS, CH. (2003): User manual BUSY-Program, EC Fifth Framework Program, Joint Research Centre of the European Commission, Ispra. 
GRÁVALOS, M.A. \& POMARES, I. (2001): "Cooperativas, desempleo y efecto refugio", REVESCO, Revista de Estudios Cooperativos, 74, 69-84.

GUERRERI, G., NAZZARO, O. \& ZEVI, A. (1992): "La economía social en Italia”. En: Monzón, J.L. \& Defourny, J. (Dirs.), Economía Social, entre economía capitalista y economía pública, Valencia, CIRIEC-España y Ministerio de Trabajo y Seguridad Social, 157-198.

HARDING, D. \& PAGAN, A. (2002): "Dissecting the Cycle: a Methodological Investigation", Journal of Monetary Economics, 49, 365-381.

HARDING, D. \& PAGAN, A. (2006): "Synchronization of cycles", Journal of Econometrics, 132 (1), 5979.

HASSLER, J. (2003): "Är Sverige I takt med Europa?". En: Ulf Jakobsson (ed.), Därför euron-tio ekonomer om den gemensamma valutan, Ekerlids Förlag, Stockholm.

LAMPEL, J., BHALLA A. \& JHA, P. (2010): "Model Growth: Do Employee-Owned Businesses deliver sustainable performance?", http://www.employeeownership.co.uk/download/MTE3

LAMPEL, J., BHALLA A. \& JHA, P. (2012): "The Employee Owned Business Model During Growth and Adversity: How Well Does it Hold Up?", European Business Review, July-August, 20-23.

LAMPEL, J., BHALLA, A. \& JHA, P. (2014): "Does governance confer organisational resilience? Evidence from UK employee owned businesses", European Management Journal, 32 (1), 66-72.

LARSSON, A., GACO, N. \& SIKSTRÖM, H. (2009): Aggregate and regional business cycle synchronization in the Nordic countries, Swedish Institute for European Policy Studies Sieps, Report $n^{0} 7$.

LEJARRIAGA, G. \& MARTíN, S. (2010): "Las sociedades laborales". En: Monzón, J.L. (Dir.), La Economía Social en España en el año 2008, CIRIEC-España, Valencia, 335-404.

MARTíN, S. \& LEJARRIAGA, G. (2011): "Las empresas de participación de trabajo asociado ante los desafíos del futuro en un contexto de crisis económica: propuestas de actuación", CIRIECEspaña, Revista de Economía Pública, Social y Cooperativa, 72, 239-261.

MCQUAID, R., CANDUELA, J., EDGELL, V. \& DUTTON, M. (2013): The growth of employee owned businesses in Scotland, Report by the Employment Research Institute, Edinburg Napier University

MELIÁN, A. \& CAMPOS, V. (2010): "Emprendedurismo y economía social como mecanismos de inserción sociolaboral en tiempos de crisis", REVESCO, Revista de Estudios Cooperativos, 100Extraordinario: La respuesta de la Economía Social ante una crisis global, 43.67.

MINTZ, I. (1969): Dating Post-War Business Cycles, methods and their applications to Western Germany, 1950-1967, Occasional Paper 107, National Bureau of Research, New York.

MONZÓN, J.L. (2012): "Las cooperativas ante la globalización: magnitudes, actividades y tendencias", Ekonomiaz, 79, 13-30. 


\section{EL EMPLEO DE LAS COOPERATIVAS. UN ANÁLISIS COMPARATIVO DE SUS FASES CÍCLICAS Y DE SU GRADO DE SINCRONIZACIÓN}

MONZÓN, J.L. \& CHAVES, R. (2012): La Economía Social en la Unión Europea, Comité Económico y Social Europeo, CIRIEC.

MOZAS, A. (Coord.) (2013): Emprendimiento y Economía Social, Monográfico de CIRIEC-España, Revista de Economía Pública, Social y Cooperativa, 78.

NEWEY, W.K. \& WEST, K.D. (1987): "A simple, positive semi-definite, heteroskedasticity and autocorrelation consistent covariance matrix", Econometrica, 55 (3), 703-708.

PARK, R., KRUSE, D. \& SESIL, J. (2004): "Does employee ownership enhance firm survival?", Advances in the Economic Analysis of Participatory and Labor-Managed Firms, Volume 8: Employee Participation, Firm Performance and Survival, 3-33.

PÉROTIN, V. (2006): "Entry, exit, and the business cycle: Are cooperatives different?", Journal of Comparative Economics, 34 (2), 295-316.

PODIVINSKY, J.M. \& GEOFF, S. (2007): "Why is labour-managed firm entry so rare?: An analysis of UK manufacturing data", Journal of Economic Behavior \& Organization, 63 (1), 177-192.

ROELANTS, B., DOVGAN, D., EUM, H. \& TERRASI, E. (2012): The resilence of the cooperative model, Report CECOP-CICOPA Europe.

ROMÁN, C. (2014): "Las cooperativas españolas y los ciclos económicos. Un análisis comparado", CIRIEC-España, Revista de Economía Pública, Social y Cooperativa, 80, 77-109.

RUSSELL, R. \& HANNEMAN, R. (1992): "Cooperatives and the business cycle: The Israeli case", Journal of Comparative Economics, 16, 701-715.

SALA, M., FARRÉ, M. \& TORRES, T. (2014): "Un análisis del comportamiento cíclico de las cooperativas y sociedades laborales españolas y de su relación con la actividad económica", REVESCO, Revista de Estudios Cooperativos, 115, 7-29.

STABER, U. (1993): "Worker cooperatives and the business cycle: are cooperatives the answer to unemployment?", The American journal of economics and sociology, 52 (2), 129-143.

TARAZONA, P. \& ALBORS, J. (2005): "La economía social y el desarrollo local", Revista Noticias del CIDEC, 45, 70-75.

WECKER, W. (1979): "Predicting turning points of a time series", Journal of business, 52 (1), 35-50.

ZARNOWITZ, V. \& OZYILDIRIM, A. (2006): "Time Series Decomposition and the Measuremen of Business Cycles, Trends and Growth Cycles", Journal of Monetary Economics, 53 (7), 1717-1739. 
